\title{
El desarrollo de competencias profesionales dentro del marco del EEES. El caso de los grados en Comunicación.
}

\author{
Isabel RuIz MORA \\ Universidad de Málaga \\ isabelruiz@uma.es \\ María Jesús RuIz MuÑoz \\ Universidad de Málaga \\ mariajesus@uma.es \\ Daniel Guerrero NAVArro \\ Universidad de Málaga \\ dguerrero@uma.es
}

Recibido: 30/07/2012

Aceptado: 29/10/2012

\begin{abstract}
Resumen
Desde el Espacio Europeo de Educación Superior (EEES) se potencia el desarrollo de destrezas profesionales desde la Universidad y esta situación se plantea como uno de los retos de este nuevo contexto educativo, caracterizado por cambios en la metodología docente y en el rol que estudiantes y docentes desempeñan en el aula. En el presente trabajo profundizamos en las posibilidades que ofrecen las herramientas docentes basadas en el aprendizaje colaborativo, para potenciar las competencias profesionales en los Estudios de Comunicación.
\end{abstract}

Palabras Clave: Estudios de Comunicación, Espacio Europeo de Educación Superior, EEES, aprendizaje colaborativo, competencias profesionales

\section{Professional skills development within the framework of the EHEA. The case of Communication studies.}

\begin{abstract}
European Higher Education Area (EHEA) enhances professional skills development from the University and this situation is seen as one of the challenges of this new educational context, characterized by changes in the teaching methodology and the role played by students and teachers. This paper goes into the potential of educational tools based on collaborative learning, in order to enhance professional skills in Communication Studies.
\end{abstract}

Keywords: Communication Studies, European Higher Education Area, EHEA, collaborative learning, professional skills

\section{Referencia normalizada}

RUIZ MORA, Isabel; RUIZ MUÑOZ, María Jesús y GUERRERO NAVARRO, Daniel (2012): “El desarrollo de competencias profesionales dentro del marco del EEES. El caso de los grados en Comunicación”. Estudios sobre el mensaje periodístico. Vol. 18, núm. especial noviembre, págs.: 839-847. Madrid, Servicio de Publicaciones de la Universidad Complutense.

Sumario: 1. Introducción. 2. Metodología. 3. Desarrollo; 3.1. Análisis DAFO. 4. Conclusiones. 5. Referencias bibliográficas.

\section{Introducción}

Peinado y Fernández (2011: 392) en su trabajo sobre la motivación de los alumnos de Grado en la elección de los estudios de Comunicación en las universidades de Madrid, 
resuelven que una de las motivaciones que argumentan los estudiantes en su elección es "la posibilidad de recibir conocimientos que les faculten en las principales técnicas de las profesiones que aspiran ejercer". Como observamos, esta razón está muy relacionada con la adquisición de competencias profesionales.

Consideremos también que en el ámbito profesional de la Comunicación, se valora especialmente la disposición y la preparación para participar en trabajo en equipo productivo, para lo cual resultan especialmente adecuadas las metodologías de aprendizaje colaborativo (AC). Estos instrumentos se revelan particularmente eficaces a la hora de fomentar en el alumnado el pensamiento crítico, los valores deontológicos y la resolución constructiva de problemas comunes, aspectos esenciales en la educación para la ciudadanía del siglo XXI (Barkley, Cross y Howell, 2007: 9). En suma, nos encontramos ante nuevas metodologías en las que los estudiantes son los protagonistas activos y el docente se convierte en catalizador del aprendizaje (Ruiz y Olmedo, 2012).

Son numerosas las técnicas y herramientas aplicadas por los docentes universitarios que tienen su base en el AC. Para la realización del presente estudio hemos realizado una selección atendiendo a su generalización en el uso y a su versatilidad a la hora de resultar aplicables al desarrollo de competencias profesionales en Comunicación.

Siguiendo estos parámetros, entre las diferentes técnicas, consideraremos el juego de rol, los debates, el método del caso (MdC), los concursos y el trabajo por proyectos. Además, nos centraremos en las que se explican a continuación, con el fin de facilitar su identificación (Imbernón y Medina, 2008: 29-31):

- Incidente crítico. Se divide la clase en pequeños grupos de trabajo que trabajan individualmente y en gran grupo, durante la puesta en común. Se presenta al grupo una situación problemática que tiene una solución y el grupo recaba información complementaria para analizar si la solución es la más adecuada. Después de esta fase, se trabaja buscando otras soluciones que conduzcan a la toma de una decisión, se debaten las propuestas en grupo reducido y se llega a una solución consensuada. Posteriormente, se ponen en común las soluciones de los distintos grupos y se reflexiona sobre el proceso y la decisión.

- 4 esquinas. El profesor selecciona cuatro respuestas u opiniones típicas y las escribe en carteles que coloca en cuatro rincones del aula. Los asistentes se pasean, leen las declaraciones y se quedan en el rincón con el que coinciden. Los grupos escogen a un portavoz y discuten su decisión, se pueden cambiar de lado si les convence otro argumento e informan después en el plenario. El profesor realiza la puesta en común.

- Puzzle. Se constituyen grupos pequeños. Se reparte la materia de modo que cada miembro del grupo reciba una parte proporcional (según dificultad y extensión). Para tratar esta parte, cada miembro se encuentra con miembros de otros grupos que han seleccionado lo mismo en un grupo nuevo (grupo experto). Hay tantos grupos expertos como partes de la materia. Cuando han acabado su trabajo, los expertos vuelven a sus grupos básicos. Entonces, los expertos juntan los elementos del puzzle; cada uno tiene el rol del profesor y explica su cono- 
cimiento de la parte de la materia. Al final, cada miembro del grupo tendría que conocer todo el material. Después, un miembro del grupo experto explica su parte del tema al plenario y el profesor matiza, amplía y expone la materia.

- Flash. Cada estudiante expresa por turnos su opinión en referencia a un problema o experiencia. No se discute hasta que todos los participantes ha hablado.

Junto con estas técnicas, incluimos una serie de herramientas que propician también el aprendizaje de habilidades profesionales a través del trabajo colaborativo. En este caso incluimos, conferencias y videoconferencias, chats, foros, wikis, blogs, redes sociales (profesionales y no profesionales) y diario de prácticas.

\section{Metodología}

Como objetivo nos planteamos profundizar en las posibilidades que ofrecen las herramientas y recursos educativos, con el fin de potenciar las competencias profesionales en los estudios de Comunicación a través del AC. Para ello, hemos seguido el siguiente diseño metodológico:

1. Identificación de las competencias profesionales de cada título de grado de Comunicación, basados en el Libro Blanco de los Títulos de Grado en Comunicación (ANECA, 2005) donde se concretan los perfiles profesionales (competencias) para los títulos de Grado de Periodismo, Comunicación Audiovisual y Publicidad y Relaciones Públicas (ANECA, 2005: 30-34).

2. Exploración de técnicas y herramientas basadas en el aprendizaje colaborativo encaminadas a fomentar el desarrollo de las competencias profesionales.

Para llevar a cabo las fases 1 y 2 hemos aplicado la técnica del análisis de contenido, como herramienta que nos permitirá la recogida, sistematización y comparación de los datos; contrastando la relación existente entre diferentes variables y la comparación de los contenidos (Igartua, 2006). De acuerdo con esta técnica, hemos procedido al diseño de una ficha de análisis para el registro de datos.

3. Propuesta de implementación basada en el AC para cada titulación, enfocada en la adquisición de competencias profesionales.

4. Evaluación de la viabilidad de la propuesta mediante una matriz DAFO.

\section{Desarrollo}

A continuación presentamos las propuestas de implementación de herramientas y técnicas de AC indicadas para alcanzar las competencias profesionales de los títulos de Comunicación.

Tabla 1. Propuesta Grado Comunicación Audiovisual

\begin{tabular}{|c|c|c|c|c|c|c|}
\hline \multicolumn{7}{|c|}{$\begin{array}{l}\text { 1. Capacidad y habilidad para planificar y gestionar los recursos humanos, presupuestarios y medios técnicos, en las } \\
\text { diversas fases de la producción audiovisual }\end{array}$} \\
\hline Juego de Rol & Conferencias & Incidente Crítico & & Foros & Chats & Diario \\
\hline RRSS espec. & Concursos & \multicolumn{3}{|l|}{ MdC } & \multicolumn{2}{|c|}{ Trabajos por proyectos } \\
\hline \multicolumn{7}{|c|}{$\begin{array}{l}\text { 2. Capacidad para crear y dirigir la puesta en escena integral de producciones audiovisuales, responsabilizándose de la } \\
\text { dirección de actores y ajustándose al guión, plan de trabajo o presupuesto }\end{array}$} \\
\hline Diario & $\mathrm{MdC}$ & Conferencias & \multicolumn{3}{|c|}{ Trabajo por proyectos } & Videoconferencias \\
\hline Foros & RRSS Esp. & Concursos & Wiki & & & Juego de rol \\
\hline
\end{tabular}


3. Capacidad para planificar y gestionar los recursos técnicos y humanos en las producciones mono-cámara y multicámara para TV, así como las técnicas y procesos de creación y realización en las distintas fases de la producción televisiva

\begin{tabular}{|l|l|l|l|l|l|}
\hline Juego de rol & Conferencias & Videoconferencias & Foros & MdC & Chats \\
\hline Diario & RRSS Esp. & Wikis & Concursos & Trabajos por proyectos \\
\hline
\end{tabular}

4. Capacidad y habilidad para gestionar técnicas y procesos de producción, registro y difusión en la organización de la producción radiofónica, discográfica y otros productos sonoros

\begin{tabular}{|l|l|l|l|l|l|}
\hline Juego de rol & Conferencias & Videoconferencias & Foros & MdC & Chats \\
\hline Diario & RRSS Esp. & Wikis & Concursos & Trabajos por proyectos
\end{tabular}

5. Capacidad y utilización de las técnicas y procesos en la organización de la producción fotográfica, así como las técnicas y procesos de creación en el campo de la imagen fija

\begin{tabular}{|l|l|l|l|l|l|}
\hline Juego de rol & Conferencias & Videoconferencias & Foros & MdC & Chats \\
\hline Diario & RRSS Esp. & Wikis & Concursos & Trabajos por proyectos \\
\hline
\end{tabular}

6. Capacidad para la utilización de las técnicas y procesos en la organización y creación en las diversas fases de la construcción de la producción multimedia y materiales interactivos

\begin{tabular}{|l|l|l|l|l|l|}
\hline Juego de rol & Conferencias & Videoconferencias & Foros & MdC & Chats \\
\hline Diario & RRSS Esp. & Wikis & Concursos & Trabajos por proyectos \\
\hline
\end{tabular}

7. Capacidad para aplicar técnicas y procedimientos de la composición de la imagen a los diferentes soportes audiovisuales, a partir del conocimiento de las leyes clásicas y de los movimientos estéticos y culturales de la historia de la imagen mediante las NNTT

\begin{tabular}{|l|l|l|l|l|}
\hline Videoconferencias & Chats & Foros & Diario & RRSS esp. \\
\hline Concurso & MdC & Trabajo por proyectos & & Conferencias \\
\hline
\end{tabular}

8. Capacidad para desarrollar mediciones vinculadas con la cantidad de luz y la calidad cromática durante el proceso de construcción de las imágenes

\begin{tabular}{|c|c|c|c|c|}
\hline Chats & Foros & Concursos & Diario & RRSS espec. \\
\hline Concursos & \multicolumn{2}{|l|}{$\mathrm{MdC}$} & \multicolumn{2}{|c|}{ Trabajo por proyectos } \\
\hline
\end{tabular}

9. Capacidad para desarrollar mediciones vinculadas con las cantidades y calidades del sonido durante el proceso de construcción del audio

\begin{tabular}{|c|c|c|c|c|c|}
\hline Concursos & Diario & RRSS espec. & Foros & \multicolumn{2}{|c|}{ Chats } \\
\hline \multicolumn{2}{|c|}{ Trabajo por proyectos } & \multicolumn{4}{|l|}{ MdC } \\
\hline \multicolumn{6}{|c|}{$\begin{array}{l}\text { 10. Capacidad para escribir con fluidez, textos, escaletas o guiones en los campos de la ficción cinematográfica, } \\
\text { televisiva, videográfica, radiofónica o multimedia }\end{array}$} \\
\hline Conferencias & Debates & Videoconferencia & Foros & Wikis & Blogs \\
\hline Concursos & Chats & Diario & RRSS esp. & Traba & or proyectos \\
\hline
\end{tabular}

11. Capacidad para analizar relatos audiovisuales, atendiendo a los parámetros básicos del análisis de obras audiovisuales

\begin{tabular}{|l|l|l|l|l|l|}
\hline Conferencias & \multicolumn{1}{|l|}{ Flash } & \multicolumn{1}{l|}{ Debates } & Chats & Foro & Blogs \\
\hline Videoconferencias & Puzzle & RRSS espec. & Trabajo por proyectos & \\
\hline
\end{tabular}

12. Capacidad para la identificación de los procesos y técnicas implicadas en la dirección y gestión de empresas audiovisuales en su estructura industrial

\begin{tabular}{|l|l|l|l|l|l|}
\hline Conferencias & Chats & Debates & Diario & Blogs & Foros \\
\hline Videoconferencias & Juego de rol & $\begin{array}{l}\text { Incidente } \\
\text { Crítico }\end{array}$ & RRSS esp. & MdC & $\begin{array}{l}\text { Trabajo por } \\
\text { proyectos }\end{array}$ \\
\hline
\end{tabular}

13. Capacidad para aplicar procesos y técnicas implicadas en la organización y gestión de recursos técnicos en cualquiera de los soportes sonoros y visuales

\begin{tabular}{|l|l|l|l|l|l|}
\hline Conferencias & Concursos & Diario & Foros & Blogs & Chats \\
\hline Videoconferencia & RRSS Esp. & MdC & Juego de rol & Trabajos por proyectos \\
\hline
\end{tabular}

14. Capacidad para aplicar técnicas y procesos de creación y difusión en el campo del diseño gráfico y de los productos multimedia e hipermedia en sus diversas fases

\begin{tabular}{|l|l|l|l|l|l|}
\hline Conferencias & Diario & Concursos & Foros & Blogs & Chats \\
\hline Juego de rol & RRSS Esp. & MdC & Videoconferencia & Trabajos por proyectos \\
\hline
\end{tabular}

15. Capacidad para aplicar técnicas y procesos de creación y recursos técnicos o humanos necesarios para el diseño de producción integral de un trabajo audiovisual

\begin{tabular}{|l|l|l|l|l|l|}
\hline Conferencias & Diario & Concursos & Foros & Blogs & Chats \\
\hline Juego de rol & RRSS Esp. & MdC & Videoconferencia & $\begin{array}{l}\text { Trabajos por } \\
\text { proyectos }\end{array}$ & Wiki \\
\hline
\end{tabular}


16. Capacidad para aplicar técnicas y procesos de producción en la organización de eventos culturales, mediante la planificación de los recursos humanos y técnicos implicados, ajustándose a un presupuesto previo

\begin{tabular}{|l|l|l|l|l|l|}
\hline Conferencias & Diario & Concursos & Foros & Blogs & Chats \\
\hline Juego de rol & RRSS Esp. & MdC & Videoconferencia & $\begin{array}{l}\text { Trabajos por } \\
\text { proyectos }\end{array}$ & Wiki \\
\hline 17. Capacide & buscar, & &
\end{tabular}

17. Capacidad para buscar, seleccionar y sistematizar cualquier tipo de documento audiovisual en una base de datos, así como su utilización en diferentes soportes audiovisuales utilizando métodos digitales

\begin{tabular}{|l|l|l|l|l|}
\hline Blogs & Foros & Wikis & Conferencias & Chats \\
\cline { 1 - 2 } & MdC & & \\
\hline
\end{tabular}

18. Capacidad para aplicar principios y funciones de la identidad visual para la creación de un manual de normas para la identidad visual corporativa de una empresa

\begin{tabular}{|l|l|l|l|l|l|}
\hline Foros & Wikis & Blogs & Chats & Concurso & Trabajo por proyectos \\
\hline
\end{tabular}

19. Capacidad para llevar a cabo el análisis de las estructuras, contenidos y estilos de la programación televisiva y radiofónica así como las distintas variables influyentes en su configuración y procesos tanto comunicativos como espectaculares por ellos generados

\begin{tabular}{|c|c|c|c|c|c|}
\hline Debate & Foros & Wikis & Blogs & Chats & Trabajo por proyectos \\
\hline
\end{tabular}

20. Capacidad para realizar la ordenación técnica de los materiales sonoros y visuales conforme a una idea utilizando las técnicas narrativas y tecnológicas necesarias para la elaboración, composición, acabado y masterización de diferentes productos audiovisuales y multimedia

\begin{tabular}{|l|l|l|l|l}
\hline Foros & Chats & Diario & RRSS esp. & Trabajos por proyectos \\
\hline
\end{tabular}

21. Capacidad para diseñar y concebir la presentación estética y técnica de la puesta en escena a través de las fuentes lumínicas y acústicas naturales o artificiales atendiendo a las características creativas y expresivas que propone el director del proyecto audiovisual

\begin{tabular}{|l|l|l|l|l|l|l|}
\hline Conferencia & Flash & Foro & Chats & Trabajo por proyectos & Diario & RRSS esp. \\
\hline
\end{tabular}

22. Capacidad para grabar señales sonoras desde cualquier fuente sonora

\begin{tabular}{|l|l|l}
\hline Diario & RRSS espec. & Trabajos por proyectos
\end{tabular}

23. Capacidad para recrear el ambiente sonoro de una producción audiovisual o multimedia atendiendo a la intención del texto y de la narración

\begin{tabular}{|l|l|l}
\hline Diario & RRSS espec. & Trabajos por proyectos \\
\hline
\end{tabular}

\section{Fuente: Elaboración propia}

\section{Tabla 2. Propuesta Grado Periodismo}

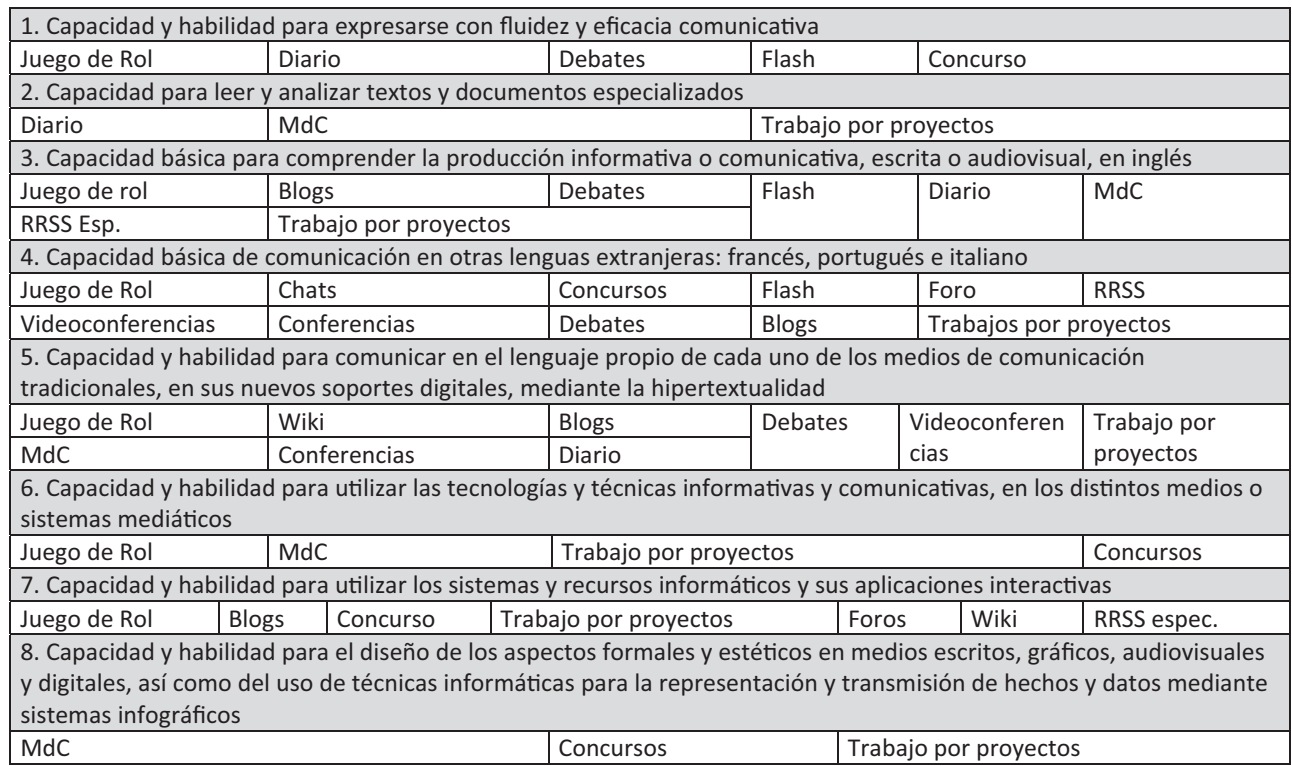




\begin{tabular}{|c|c|c|c|c|c|}
\hline \multicolumn{6}{|c|}{ eación, planificación y eje } \\
\hline Juego de Rol & Blogs & RRSS espec. & Foros & Chats & \multirow[t]{2}{*}{$\mathrm{MdC}$} \\
\hline Wiki & Diario & Concursos & \multicolumn{2}{|c|}{ Trabajo por proyectos } & \\
\hline \multicolumn{6}{|c|}{$\begin{array}{l}\text { 10. Capacidad y habilidad para el desempeño de las principales tareas periodísticas, desarrolladas a través de áreas } \\
\text { temáticas, aplicando géneros y procedimientos periodísticos }\end{array}$} \\
\hline Juego de Rol & Blogs & RRSS espec. & Debates & Conferencias & Foro \\
\hline $\mathrm{MdC}$ & Videoconferencias & Conferencias & Concursos & \multicolumn{2}{|c|}{ Trabajos por proyectos } \\
\hline \multicolumn{6}{|c|}{$\begin{array}{l}\text { 11. Capacidad y habilidad para buscar, seleccionar y jerarquizar cualquier tipo de fuente o documento de utilidad para } \\
\text { la elaboración y procesamiento de información, así como para su aprovechamiento comunicativo }\end{array}$} \\
\hline \multicolumn{2}{|c|}{$\mathrm{MdC}$} & & \multicolumn{2}{|c|}{ Trabajo por proyectos } & \\
\hline \multicolumn{6}{|c|}{$\begin{array}{l}\text { 12. Capacidad y habilidad para recuperar, organizar, analizar y procesar información y comunicación con la finalidad de } \\
\text { ser difundida, servida o tratada para usos privados o colectivos a través de diversos medios y soportes }\end{array}$} \\
\hline \multicolumn{2}{|c|}{$\mathrm{MdC}$} & & \multicolumn{2}{|c|}{ Trabajo por proyectos } & \\
\hline \multicolumn{6}{|c|}{$\begin{array}{l}\text { 13. Comprensión de los datos y de las operaciones matemáticas efectuadas con algunos de ellos de uso corriente en } \\
\text { los medios de comunicación y capacidad y habilidad para saber utilizar datos y estadísticas de manera correcta y } \\
\text { comprensible para la divulgación }\end{array}$} \\
\hline \multicolumn{2}{|c|}{$\mathrm{MdC}$} & & \multicolumn{3}{|c|}{ Trabajo por proyectos } \\
\hline \multicolumn{6}{|c|}{$\begin{array}{l}\text { 14. Capacidad y habilidad de exponer razonadamente ideas, a partir de los fundamentos de la retórica y de las } \\
\text { aportaciones de las nuevas teorías de la argumentación, así como de las técnicas comunicativas aplicadas a la } \\
\text { persuasión }\end{array}$} \\
\hline Juego de Rol & Videoconferencias & Conferencias & $\mathrm{MdC}$ & Flash & \\
\hline Blogs & Puzzle & Debates & RRSS & \multicolumn{2}{|l|}{ RRSS espec. } \\
\hline \multicolumn{6}{|c|}{$\begin{array}{l}\text { 15. Capacidad de experimentar e innovar mediante el conocimiento y uso de técnicas y métodos aplicados a los } \\
\text { procesos de mejora de la calidad y de auto evaluación, así como habilidades para el aprendizaje autónomo, la } \\
\text { adaptación a los cambios y la superación rutinaria mediante la creatividad }\end{array}$} \\
\hline Juego de Rol & Incidente Crítico & Foros & Debates & \multirow{2}{*}{\multicolumn{2}{|c|}{ Trabajo por proyectos }} \\
\hline Flash & Diario & \multicolumn{2}{|l|}{$\mathrm{MdC}$} & & \\
\hline
\end{tabular}

\section{Fuente: Elaboración propia}

\section{Tabla 3. Propuesta Grado Publicidad y Relaciones Públicas}

\begin{tabular}{|c|c|c|c|c|c|}
\hline \multirow{2}{*}{\multicolumn{2}{|c|}{$\begin{array}{l}\text { 1. Capacidad y habilidad para ej } \\
\text { asistiéndole de manera continua } \\
\text { MdC }\end{array}$}} & \multirow{3}{*}{$\begin{array}{l}\text { ercer com } \\
\text { ada antes } \\
\text { Puzzle } \\
\text { Chats } \\
\end{array}$} & \multirow{2}{*}{\multicolumn{2}{|c|}{$\begin{array}{l}\text { spués de la rea } \\
\text { Juego de Rol }\end{array}$}} & $\begin{array}{l}\text { cliente de la agencia, } \\
\text { s de comunicación }\end{array}$ \\
\hline & & & & & \multirow{2}{*}{$\begin{array}{l}4 \text { esquinas } \\
\text { RRSS Esp. }\end{array}$} \\
\hline Wiki & Blogs & & Diario & Foros & \\
\hline \multicolumn{2}{|c|}{ Videoconferencias } & \multicolumn{2}{|c|}{ Incidente Crítico } & \multicolumn{2}{|l|}{ Debates } \\
\hline \multicolumn{6}{|c|}{ 2. Capacidad y habilidad para responsabilizarse del área de comunicación de una organización } \\
\hline Wiki & Blogs & Chats & Diario & RRSS Esp. & Foros \\
\hline \multicolumn{2}{|l|}{4 esquinas } & \multicolumn{2}{|l|}{ MdC } & \multicolumn{2}{|c|}{ Videoconferencias } \\
\hline \multicolumn{2}{|c|}{ Juego de Rol } & Puzzle & Debates & \multicolumn{2}{|c|}{ Incidente Crítico } \\
\hline \multicolumn{6}{|c|}{ 3. Capacidad y habilidad para establecer el plan de comunicación } \\
\hline Diario & Puzzle & Foros & Wiki & Blogs & Chats \\
\hline \multicolumn{2}{|c|}{ Incidente Crítico } & RRSS Esp. & $\mathrm{MdC}$ & & 4 esquinas \\
\hline \multicolumn{6}{|c|}{$\begin{array}{l}\text { 4. Capacidad y habilidad para el ejercicio liberal de la profesión y la docencia prestando asesoramiento a agencias d } \\
\text { comunicación o a sus clientes en materias vinculadas con la comunicación }\end{array}$} \\
\hline Chats & Flash & Puzzle & Wiki & \multicolumn{2}{|c|}{ Trabajo por proyectos } \\
\hline 4 esquinas & Blogs & Concursos & $\mathrm{MdC}$ & Debates & Videoconferencias \\
\hline \multicolumn{2}{|c|}{ Incidente Crítico } & Juego de Rol & Foros & Diario & RRSS Esp. \\
\hline \multicolumn{6}{|c|}{$\begin{array}{l}\text { 5. Capacidad relacional y de indagación para establecer una interacción fluida y competente entre el anunciante y la } \\
\text { agencia }\end{array}$} \\
\hline Puzzle & Concursos & \multicolumn{2}{|c|}{ Trabajo por proyectos } & \multicolumn{2}{|c|}{ Método del Caso } \\
\hline Wiki & Flash & Debates & Foros & RRSS Esp. & Juego de Rol \\
\hline \multicolumn{2}{|c|}{ Videoconferencias } & Chats & Diario & $\mathrm{MdC}$ & Blogs \\
\hline
\end{tabular}




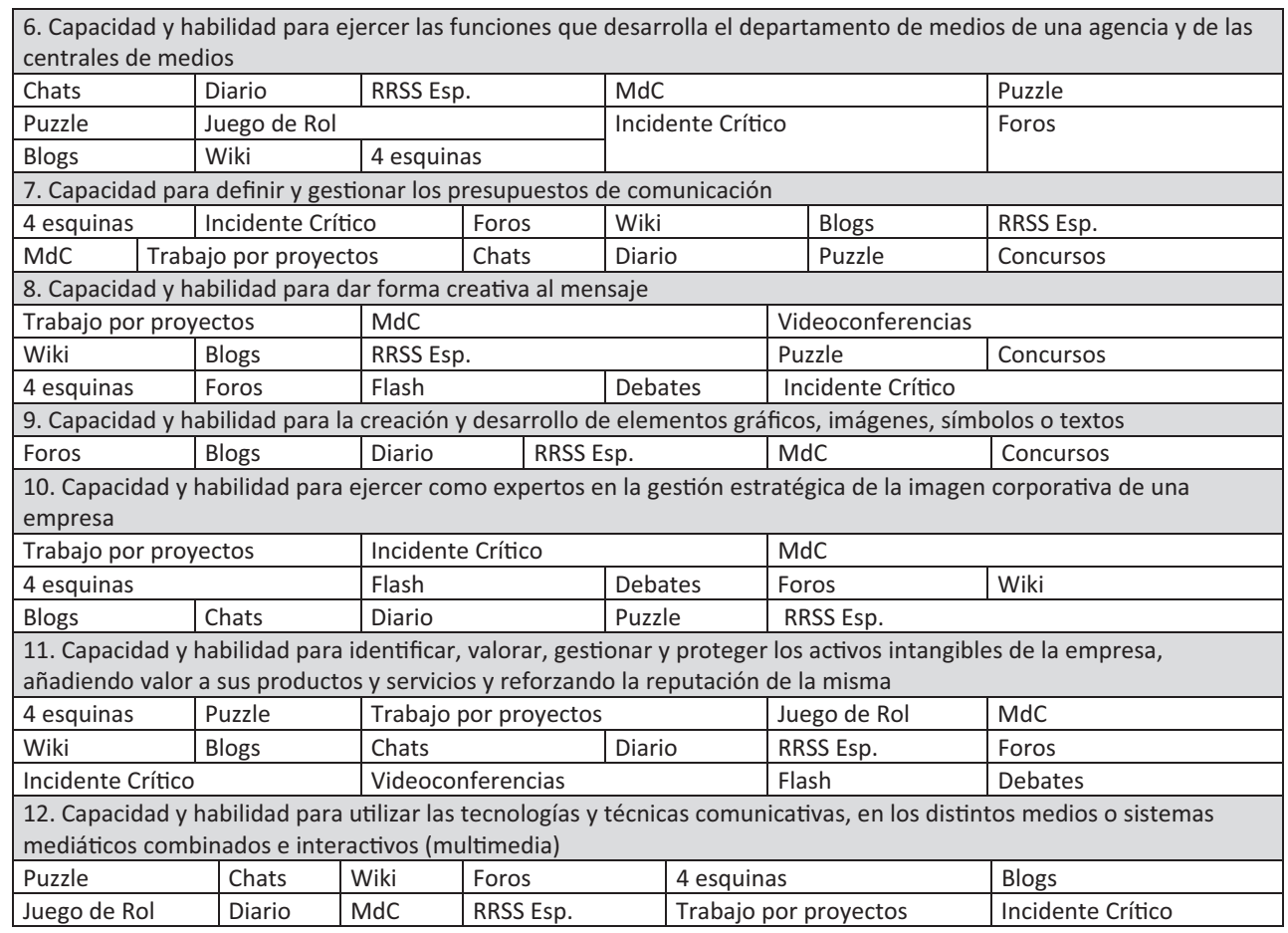

Fuente: Elaboración propia

\subsection{Análisis DAFO}

Mediante la matriz DAFO hemos valorado las propuestas presentadas para alcanzar las competencias profesionales en los estudios de Comunicación mediante el AC. Para ello, nos hemos aproximado a las debilidades y fortalezas (centradas en la propuesta en sí y en el docente) y a las amenazas y oportunidades (enfocadas en el entorno y los estudiantes como receptores de las propuestas) de las propuestas.

\section{Tabla 4. Matriz DAFO}

\begin{tabular}{|l|l|}
\hline \multicolumn{1}{|c|}{ Debilidades } & \multicolumn{1}{c|}{ Fortalezas } \\
-Desconocimiento de técnicas y herramientas por parte & -Alguna formación previa del docente sobre AC \\
del docente & -Motivación del docente \\
-Dificultad para la evaluación & -Cambio de rol: el docente provoca el aprendizaje \\
-Dificultad para conciliar tiempo de trabajo con & -No es clase magistral \\
planificación & -Se trabajan conocimientos teóricos-prácticos \\
-Inexperiencia en el cálculo de las horas necesarias para & -Aprovechamiento de las actividades \\
desarrollar actividades según créditos ECTS & complementarias para activar nuevas técnicas \\
-Los cambios en la asignación docente del profesorado & -Incentivación de la innovación educativa en el \\
pueden ser una traba para el perfeccionamiento de & sistema actual de enseñanza-aprendizaje \\
técnicas y herramientas & -Promoción de intercambio de experiencias entre \\
-Coordinación de metodologías y herramientas & docentes universitarios. \\
aplicadas en asignaturas compartidas por varios & \\
docentes & \\
\hline
\end{tabular}




\begin{tabular}{|c|c|}
\hline \begin{tabular}{l}
\multicolumn{1}{c}{ Amenazas } \\
-Infraestructuras no adecuadas al EEES \\
-Estudiantes poco motivados \\
-Prejuicios sobre el trabajo en grupo \\
-Desconocimiento o dificultad del docente para \\
reproducir circunstancias que simulen casos reales \\
-Inexistencia de actores que estudien y vinculen a través \\
de indicios o fuentes la adecuación de los perfiles o \\
competencias a la situación real de su profesión \\
-Escasez de lazos de colaboración entre los agentes \\
(académico y profesional)
\end{tabular} & \begin{tabular}{l}
\multicolumn{1}{c}{ Oportunidades } \\
-Acercamiento el mundo profesional a la \\
Universidad \\
-Trabajo en equipo \\
-Trabajo con casos reales \\
-Cambio de rol: el estudiante participa en su \\
aprendizaje \\
-Relación cercana estudiante/docente \\
-Estudiantes familiarizados con NNTT \\
-Desarrollo de competencias profesionales \\
-Pensamiento crítico \\
-Fomento del interés por la investigación e \\
innovación \\
-Sentimiento de solidaridad (pertenencia) \\
-Resultados tangibles
\end{tabular} \\
\hline
\end{tabular}

Fuente: Elaboración propia

\section{Conclusiones}

El trabajo desarrollado nos ha permitido identificar las técnicas y herramientas que propician la adquisición de competencias profesionales en Comunicación trabajando con el aprendizaje colaborativo. Esta identificación puede provocar una reorientación del trabajo en el aula a fin de alcanzar las deseadas competencias profesionales.

Profundizar en el conocimiento de las debilidades y amenazas del aprendizaje colaborativo nos orienta en el diseño y planteamiento de la actividad en el aula, igualmente nos permite conocer con antelación los posibles problemas a los que nos podremos enfrentar, por lo que disminuimos el grado de fracaso de la actividad, de insatisfacción en el alumnado y de frustración del docente. En general, estas dificultades se relacionan con carencias relacionadas con la falta de formación y/o experiencia del profesorado, limitaciones del tiempo y/o recursos técnicos disponibles y escasez de motivación del alumnado. Sin embargo, el análisis de fortalezas y oportunidades, revela que el aprendizaje colaborativo no sólo sirve para fomentar el interés y la implicación del alumnado sino que también resulta útil para trabajar otras competencias transversales que se relacionan con alimentar su capacidad de análisis y promover el trabajo con nuevas tecnologías. Mediante el trabajo colaborativo, desarrollamos entre los estudiantes algo más que competencias profesionales, ya que provocamos en ellos un pensamiento más crítico y solidario.

Por otro lado, los modelos propuestos permitirán a los docentes distinguir y seleccionar las técnicas y herramientas más adecuadas para el desarrollo de cada destreza profesional. Además, el estudio realizado revela que las técnicas y herramientas con un mayor índice de adecuación para desarrollar competencias profesionales son aquellas que ofrecen un campo de trabajo más global y similar de la realidad profesional: juego de rol, método del caso, trabajo por proyectos y redes sociales especializadas.

Por todo lo expuesto, este trabajo no queda aquí agotado ni mucho menos, los resultados obtenidos nos invitan a seguir explorando las posibilidades detectadas con motivo del trabajo desarrollado. Cabe destacar que, entre los horizontes que se vislumbran, resulta de especial interés la línea relacionada con el desarrollo e implementación de técnicas y herramientas de $\mathrm{AC}$ concebidas específicamente para trabajar 
competencias profesionales en las titulaciones de Comunicación, como son cinefórums, videofórums, performances o lipdubs.

\section{Referencias Bibliográficas}

AGENCIA NACIONAL DE EVALUACIÓN DE LA CALIDAD Y ACREDITACIÓN (ANECA, 2005): Libro Blanco de los Títulos de Grado en Comunicación. http://www.aneca.es/var/media/150336/libroblanco_comunicacion_def.pdf.

BARKLEY, Elizabeth; CROSS, K. Patricia \& HOWĒLL MAYOR, Claire (2007): Técnicas de aprendizaje colaborativo. Madrid, Ministerio de Educación y Ciencia/ Morata.

IGARTUA PEROSANZ, Juan José (2006): Métodos Cuantitativos de Investigación en Comunicación. Barcelona, Bosch Comunicación.

IMBERNÓN MUÑOZ, Francesc \& MEDINA MOYA, José Luis (2008): Metodología participativa en el aula universitaria. La participación del alumnado. Cuadernos de docencia universitaria 04. Barcelona, ICE y Ediciones Octaedro.

PEINADO MIGUEL, Fernando \& FERNÁNDEZ SANDE, Manuel (2011): "Reflexión sobre la motivación de los alumnos de Grado en la elección de estudios de comunicación en las universidades de Madrid", en Estudios sobre el Mensaje Periodístico, vol. 17, $\mathrm{n}^{\circ}$ 2. Madrid, Servicio de Publicaciones de la Editorial Complutense, pp. 383-400.

RUIZ MORA, Isabel \& OLMEDO SALAR, Silvia (2012): "Desarrollo de competencias profesionales en el espacio europeo de educación superior (EEES). Nuevas metodologías en los estudios de comunicación", en Revista de Comunicación Vivat Academia, $\mathrm{n}^{\mathrm{o}} 117 \mathrm{http} / /$ www.ucm.es/info/vivataca/numeros/n117E/PDFs/Varios32.pdf

\section{Isabel RUIZ MORA}

\section{María JESÚS RUIZ MUÑOZ}

\section{Daniel GUERRERO NAVARRO}

Docentes e investigadores de la Facultad de Ciencias de la Comunicación de la Universidad de Málaga 